\title{
Sexualidade e Surdez: uma Revisão Sistemática
}

\author{
Valéria Maria Azevedo Guimarães ${ }^{1}$ \\ ${ }^{1}$ Universidade Federal de Sergipe, SE, Brasil.
}

\author{
Joilson Pereira da Silva ${ }^{1}$ \\ ${ }^{1}$ Universidade Federal de Sergipe, SE, Brasil.
}

\begin{abstract}
Resumo: A existência de mitos e tabus que envolvem a sexualidade pode dificultar a disseminação de informações adequadas para a sociedade. Sabe-se que a população surda, por não ter os mesmos meios de aquisição de informações dos ouvintes, lida com limitações no acesso a essa temática. O objetivo desta pesquisa foi realizar uma revisão sistemática de artigos científicos sobre sexualidade e surdez. Para isso, no período de setembro a novembro de 2017, foi feita uma busca utilizando os descritores sexualidade, surdez, surdo e deficiente auditivo, nas línguas portuguesa, inglesa e espanhola, em cinco bases de dados. A busca teve o período delimitado entre 2000 e 2017, e o resultado final selecionou quinze artigos. A sexualidade por meio de experiências sexuais, mitos e relacionamentos e a violência e/ ou abuso sexual foram os assuntos com maior prevalência. A questão da homossexualidade foi menos evidente nas produções científicas. A revisão mostrou que o conjunto de estudos avaliados retrata a sexualidade de forma abrangente e com temas atuais. Além disso, são escassos os estudos científicos referentes a sexualidade e surdez, sendo necessários mais estudos com a participação das pessoas surdas no intuito de compreendê-las, orientá-las e informá-las sobre o assunto em questão.
\end{abstract}

Palavras-chave: Sexualidade, Surdez, Deficiente Auditivo, Educação Sexual, Saúde Sexual.

\section{Sexuality and Deafness: a Systematic Review}

\begin{abstract}
The existence of myths and taboos about sexuality may lead to challenges in spreading adequate information to society. It is a known fact that the deaf population faces a lack of information about this theme caused by the absence of means to acquire the same information that listeners have. Thus, this systematic review of scientific articles about sexuality and deafness was conducted. Between September 2017 and November 2017, we searched in five databases (SciELO, PePSIC, LILACS, PsycINFO and Scopus) for the descriptors sexuality, deafness, deaf and hearing impaired, in Portuguese, English and Spanish, for the period between 2000-2017, rendering 15 articles. Sexuality through sexual experiences, mythos and relationships, as well as violence and/or sexual abuse were the subjects with highest prevalence. However, the matter of homosexuality was less evident in scientific productions. The review showed that the set of evaluated studies portrayed sexuality in an embracing manner and involved current themes. In addition, there is a lack of scientific articles about sexuality and deafness, and so more studies with deaf people to understandd them, guide them and inform them about the subject in question are required.
\end{abstract}

Keywords: Sexuality, Deafness, Hearing Impaired, Sexual Education, Sexual Health. 


\title{
Sexualidad y Sordera: una Revisión Sistemática
}

\begin{abstract}
Resumen: La existencia de mitos y tabúes que envuelven la sexualidad puede dificultar la diseminación de informaciones adecuadas para la sociedad. Se sabe que la población sorda por no poseer los mismos medios de adquisición de informaciones que los oyentes pasa por limitaciones de informaciones sobre esa temática. El objetivo de esta investigación fue realizar una revisión sistemática de artículos científicos sobre sexualidad y sordera. Para ello, se realizó una búsqueda de septiembre a noviembre de 2017 utilizando los descriptores "sexualidad", "sordera", "sordos y deficiente auditivo", en portugués, inglés y español, en cinco bases de datos. La búsqueda tuvo el período delimitado entre 2000-2017 y en el resultado final se seleccionó 15 artículos. La sexualidad a través de las experiencias sexuales, de los mitos y relaciones y la violencia y/o abuso sexual fueron los asuntos con mayor prevalencia. Pero el tema de la homosexualidad fue el menos evidente en las producciones científicas. La revisión mostró que el conjunto de los estudios evaluados retratan la sexualidad de forma integral y con temas actuales. Además, hay escasez de estudios científicos referentes a la sexualidad y sordera y son necesarios más estudios con la participación de las personas sordas con el fin de comprenderlas, orientarlas e informarlas sobre el asunto en cuestión.
\end{abstract}

Palabras clave: Sexualidad, Sordera, Deficiente Auditivo, Educación Sexual, Salud Sexual.

\section{Introdução}

No mundo existem cerca de 466 milhões de pessoas com deficiência auditiva, sendo que 34 milhões são crianças (World Health Organization, 2013). No Brasil, de acordo com o Censo de 2010, há 45 milhões de pessoas com algum tipo de deficiência, e destas 7,6\% são totalmente surdas (Oliveira, 2012). Os surdos vivem em uma sociedade com predominância de ouvintes e deparam com dificuldades diárias de comunicação, estigmas e exclusão. Por conta disso, dificuldades e barreiras linguísticas e culturais são observadas, e torna-se necessário compreender que surdos e deficientes auditivos não possuem os mesmos meios de aquisição de informações dos ouvintes (Luckner \& Gonzales, 1993).

Batista e Reis (2011) investigaram a comunicação entre estudantes surdos e pais ouvintes e perceberam que a ausência do conhecimento da Língua Brasileira de Sinais (Libras) por parte dos familiares foi considerada um obstáculo para a comunicação e, consequentemente, dificultou o acompanhamento no processo educacional. Além disso, o domínio da Libras por familiares auxilia na aprendizagem e na consolidação da identidade linguística e cultural da prole. Estudiosos do assunto ressaltaram que o desconhecimento e/ou a não fluência em Libras no ambiente familiar ocasiona restrições no diálogo e nas orientações e aumento das dúvidas dos surdos sobre o tema da sexualidade (Bisol, 2008; Lebedeff, 2010; Ribeiro, 2011).

A barreira linguística também é percebida por surdos em locais públicos ou no contato com profissionais da saúde, pois geralmente há limitação da autonomia e diminuição da privacidade, ocasionada pela necessidade de acompanhamento (Marin \& Góes, 2006). Assim, a comunicação prejudicada entre o surdo e o ouvinte reduz o conhecimento do surdo sobre diversos temas sociais, inclusive a respeito da saúde, e isso pode prejudicar seu diagnóstico e tratamento (Chaveiro, Barbosa, \& Porto, 2008; Dizeu \& Caporali, 2005).

Entretanto, para amenizar esse obstáculo da comunicação, Labarre (1998) e Sullivan, Scanlan, Brookhouser e Schulte (1992) descreveram o tratamento psicológico com crianças surdas que foram abusadas sexualmente, e assinalaram aspectos importantes para o profissional realizar o atendimento a pessoa surda: a necessidade de saber se comunicar e conhecer a cultura do surdo; usar da criatividade para adaptar as técnicas, deixando-as mais visuais e concretas; e aumentar o tempo das sessões.

Em revisão de literatura acerca do abuso em crianças com deficiência constatou-se que aquelas com distúrbios da fala e do idioma, deficiências auditivas e dificuldades de aprendizagem representam 
um grupo com maior risco de sofrer abuso físico ou sexual (Sullivan \& Knutson, 1998). Além disso, Yu et al. (2017) examinaram o conhecimento de prevenção da criança sexualmente abusada e suas habilidades de autoproteção e destacaram que crianças com deficiência auditiva precisam aprender como recusar solicitações ou demandas inapropriadas para que consigam identificar o abusador. Para isso, os autores afirmaram que é necessário promover o aumento do nível de conhecimento dos pais e incentivar a interação pai-filho para que a base do conhecimento das crianças sobre as capacidades de autoproteção seja melhorada. Adicionalmente, Getch, Branca, FitzGerald e Fitz-Gerald (2001) e Bandarra (2013) pontuaram a necessidade dos pais e professores na educação sexual dos jovens.

Bisol, Sperb, Brewer, Kato e Shor-Posner (2008) investigaram o conhecimento dos adolescentes surdos e ouvintes sobre o HIV/aids e examinaram as atitudes e comportamentos desses jovens relacionados à saúde. Com isso, foi identificada uma diferença significativa no conhecimento do HIV/aids entre ouvintes e surdos, demonstrando que os primeiros poussuem mais informações sobre essas doenças. Estudos semelhantes (Baker-Duncan, Dancer, Gentry, Highly, \& Gibson, 1997; Doyle, 1995; Kennedy \& Buchhoiz, 1995; Luckner \& Gonzales, 1993) abordaram o HIV/ aids em pessoas surdas, por ser uma doença que não possui cura e pelo fato de educação e prevenção serem recursos que podem dirimir a propagação da aids. Destarte, omitir informações sobre a sexualidade pode tornar os surdos vulneráveis a comportamentos de risco (infecções sexualmente transmissíveis ISTs e gravidez não programada), a violência e abuso sexual e ao preconceito por conta de sua orientação sexual (Luckner \& Gonzales, 1993; Sousa \& Moleiro, 2015; Sousa \& Pagliuca, 2001).

Bento (2005) afirmou que os surdos possuem conhecimento simplista sobre a sexualidade e que os mitos e crenças são presentes em seus discursos. Ademais, foi observada a inadequação das informações sobre essa temática e ressaltada a relevância de uma ação/intervenção educativa efetiva com o intuito de garantir que a pessoa surda tenha uma escolha responsável sobre sua saúde sexual e reprodutiva. Segundo Job (2004), alguns aspectos podem influenciar na falta de conhecimento dos surdos sobre a sexualidade: a) as poucas oportunidades para obterem informações; b) a resistência dos familiares em promover a educação sexual; e c) o desconhecimento de colegas sobre a temática e o repasse de informações errôneas sobre a questão.

A sexualidade está presente em todas as fases do desenvolvimento do ser humano, e a vivência dela dependerá dos valores e das práticas sociais de cada indivíduo inserido em culturas distintas (Ribeiro, 2011). Adicionalmente, o conceito de sexualidade é construído historicamente e se encontra em constante expansão. Por conta da amplitude do conceito, ele abrange temáticas como "gênero, identidade sexual, orientação sexual, erotismo, envolvimento emocional, amor e reprodução" (Castro, Abramovay, \& Silva, 2004, p. 29). Ademais, Aquino (1997) acrescenta a necessidade de discutir a temática, pois auxilia na formação da identidade do sujeito. Nesse sentido, o objetivo desta pesquisa foi realizar uma revisão sistemática de artigos científicos sobre sexualidade e surdez. Cabe salientar que o conhecimento acerca dos estudos referentes a essa temática promoverá a identificação de lacunas e auxiliará a novas perspectivas de investigação.

\section{Método}

Foi realizado um levantamento bibliográfico em cinco bases de dados: SciELO, PePSIC, Lilacs, PsycINFO e Scopus. As buscas nesses bancos ocorreram nos meses de setembro, outubro e novembro de 2017. Os descritores utilizados foram selecionados a partir da Terminologia em Psicologia da Biblioteca Virtual de Psicologia (BVS - PSI Brasil), e foi empregado o operador booleano AND entre os termos em três diferentes línguas, com a seguinte estratégia de busca: a) em português: sexualidade AND surdez; sexualidade AND surdo; sexualidade AND deficiente auditivo; b) em inglês: sexuality AND deafness; sexuality AND deaf; sexuality AND hearing disorders; c) em espanhol: sexualidad AND sordera; sexualidad AND sordo; sexualidad AND deficiente auricular.

Os critérios de inclusão foram: a) versar sobre a surdez com foco na sexualidade; b) ter na amostra pessoas surdas; c) ter o português, inglês ou o espanhol como idioma; e d) ser publicado no período de 2000 a novembro de 2017. Os critérios de exclusão foram: a) ter na amostra pessoas com implante coclear; b) publicações duplicadas; c) publicações em outros formatos que não fossem artigo; d) artigos de revisão; e e) não disponibilização do resumo e do texto completo por via on-line e de forma gratuita. 
O procedimento de busca inicial ocorreu com a leitura do título, das palavras-chave e do resumo dos artigos. Após seleção dos artigos que cumpriam os critérios de inclusão, as publicações que se repetiram entre as bases e na própria base, bem como os artigos com acesso bloqueado ou pagos, foram excluídas. Posteriormente, foi feita a leitura na íntegra dos artigos selecionados para realização da análise. Para os procedimentos de busca, seleção e análise, foram designados três juízes independentes. Em caso de discordância quanto a sua inclusão, os juízes discutiram novamente $\mathrm{o}$ artigo até obterem um parecer final.

Os artigos selecionados após a leitura na íntegra foram submetidos a folha de codificação composta por 18 itens: 1) Número de identidade do artigo; 2) Termo encontrado; 3) Título; 4) Banco de dados; 5) Endereço na internet; 6) Autores; 7) Ano da publicação; 8) Periódico; 9) Idioma do texto; 10) PalavrasChave; 11) Tipo de publicação; 12) Filiação dos autores; 13) Objetivo do trabalho; 14) Participantes;
15) Instrumento e/ou técnica de pesquisa; 16) Procedimento; 17) Resultados; e 18) Problemas/ limitações encontrados nos resultados da pesquisa.

\section{Resultados}

A busca nas bases de dados consultadas resultou em 124 artigos (SciELO - 0 artigos; PePSIC - 6 artigos; Lilacs - 9 artigos; PsycINFO - 74 artigos; e Scopus - 35 artigos), sendo que 5 foram excluídos após a leitura de seus títulos, resumos e palavras-chave, pois não atendiam aos critérios de inclusão e/ou atendiam aos critérios de exclusão. Após a primeira exclusão, restaram 119 artigos, e destes foram excluídos 77 por se repetirem entre as bases de dados, permanecendo 42 publicações para análise. Em seguida, foram excluídos 27 artigos pagos ou que não permitiam seu acesso gratuito e/ou on-line. Por fim, todos os 15 artigos remanescentes foram lidos na íntegra e compuseram o resultado final da busca, conforme Figura 1.

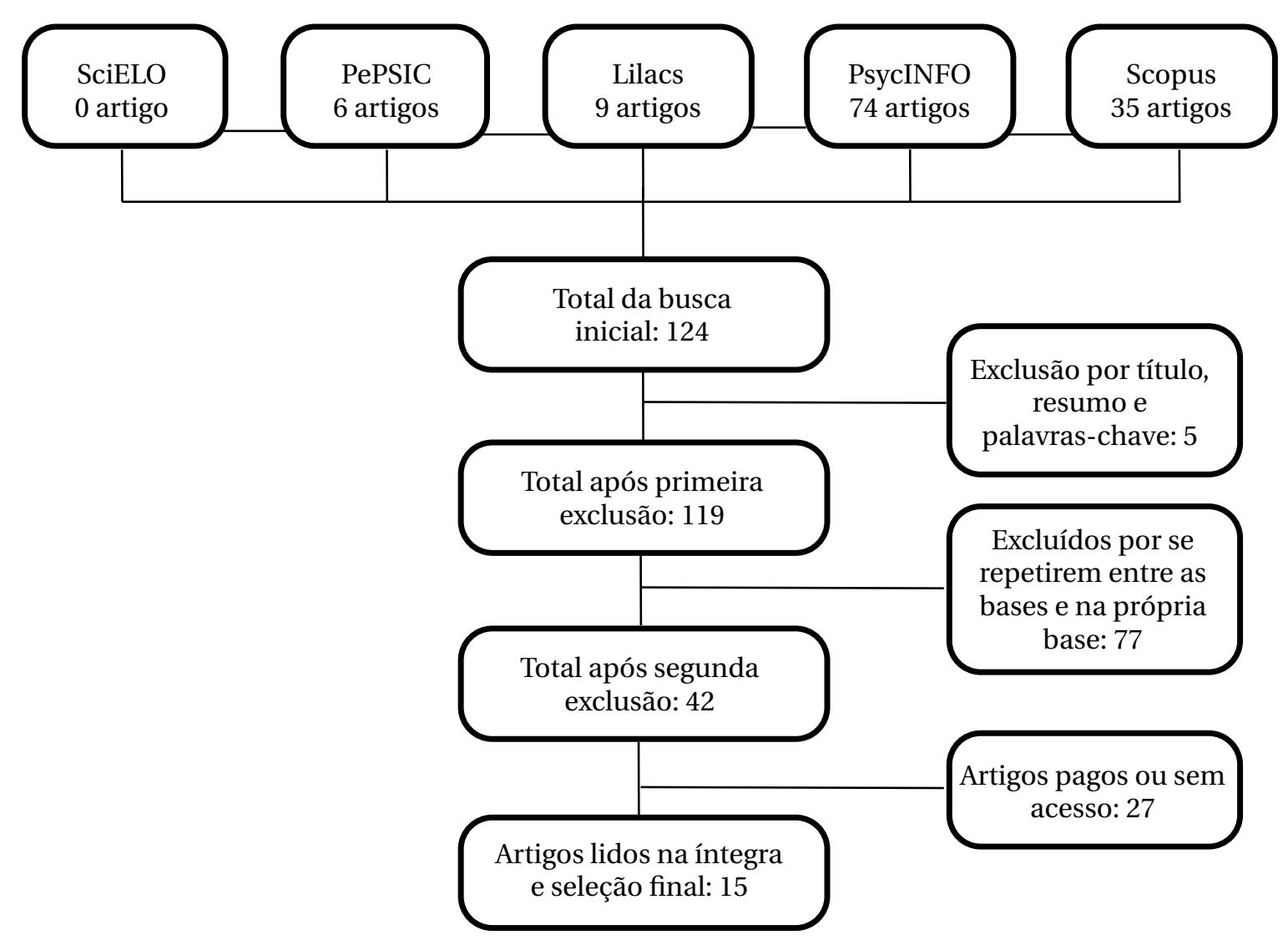

Figura 1

Fluxograma do processo de seleção dos artigos. 
Do total de artigos analisados, 13 foram redigidos na língua inglesa, um na língua portuguesa e um em espanhol. Embora o maior número de estudos seja em inglês, quatro foram conduzidos em países onde o inglês não era o principal idioma. $\mathrm{O}$ país que mais se destacou nas produções científicas foi os Estados Unidos da América $(\mathrm{n}=7)$, seguido do Brasil $(\mathrm{n}=2)$. Os demais países (Colômbia, Dinamarca, Alemanha, Nigéria, Filipinas e Taiwan) tiveram um artigo publicado. Quanto à abordagem do estudo, verificou-se que o método quantitativo foi o mais utilizado $(n=6)$, porém em cinco artigos não constava a abordagem dos estudos, três eram qualitativos e um usou o método misto. Em relação às instituições, havia cinco estudos provenientes da primeira universidade para surdos do mundo, a Universidade Gallaudet.

Ademais, observaram-se diferentes instrumentos e/ou técnicas de pesquisa que foram utilizados por pesquisadores para as coletas de dados: entrevistas, escalas e subescalas (sobre o bem-estar psicológico, satisfação sexual e resolução de conflitos e vitimização), diferentes tipos de questionários (demográfico, sobre a sexualidade, comportamento de risco e o questionário não padronizado do Centro Federal para Educação em Saúde), grupo focal e o uso de imagens, desenho e vídeo. Houve repetição dos instrumentos e/ou técnicas entre os artigos: entrevistas $(n=3)$, grupo focal $(n=2)$, questionário demográfico $(n=3)$, Escala de Táticas de Conflito (CTS), a Escala Revisada de Táticas de Conflitos (CTS2) ( $\mathrm{n}=3)$ - neste caso, o que diferenciou uma escala da outra foi a revisão feita na escala - e subescalas de vitimização $(n=2)$.

Além disso, observou-se que os anos com mais publicações foram 2011, 2012 e 2014, com três artigos em cada ano, seguido de 2015 e2017, com dois estudos, e 2009 e 2016, com um pesquisa em cada, conforme a Figura 2. Os periódicos com maior frequência de produções foram Sexuality and Disability $(\mathrm{n}=5)$, The Journal of Deaf Studies and Deaf Education e Violence Against Women, com duas publicações em cada.Os periódicos restantes (Pensamiento Psicológico, Temas em Psicologia, Psychology, Sexuality \& Culture, Revista da Sociedade Brasileira de Medicina Tropical e Journal of Interpersonal Violence) tiveram uma publicação.

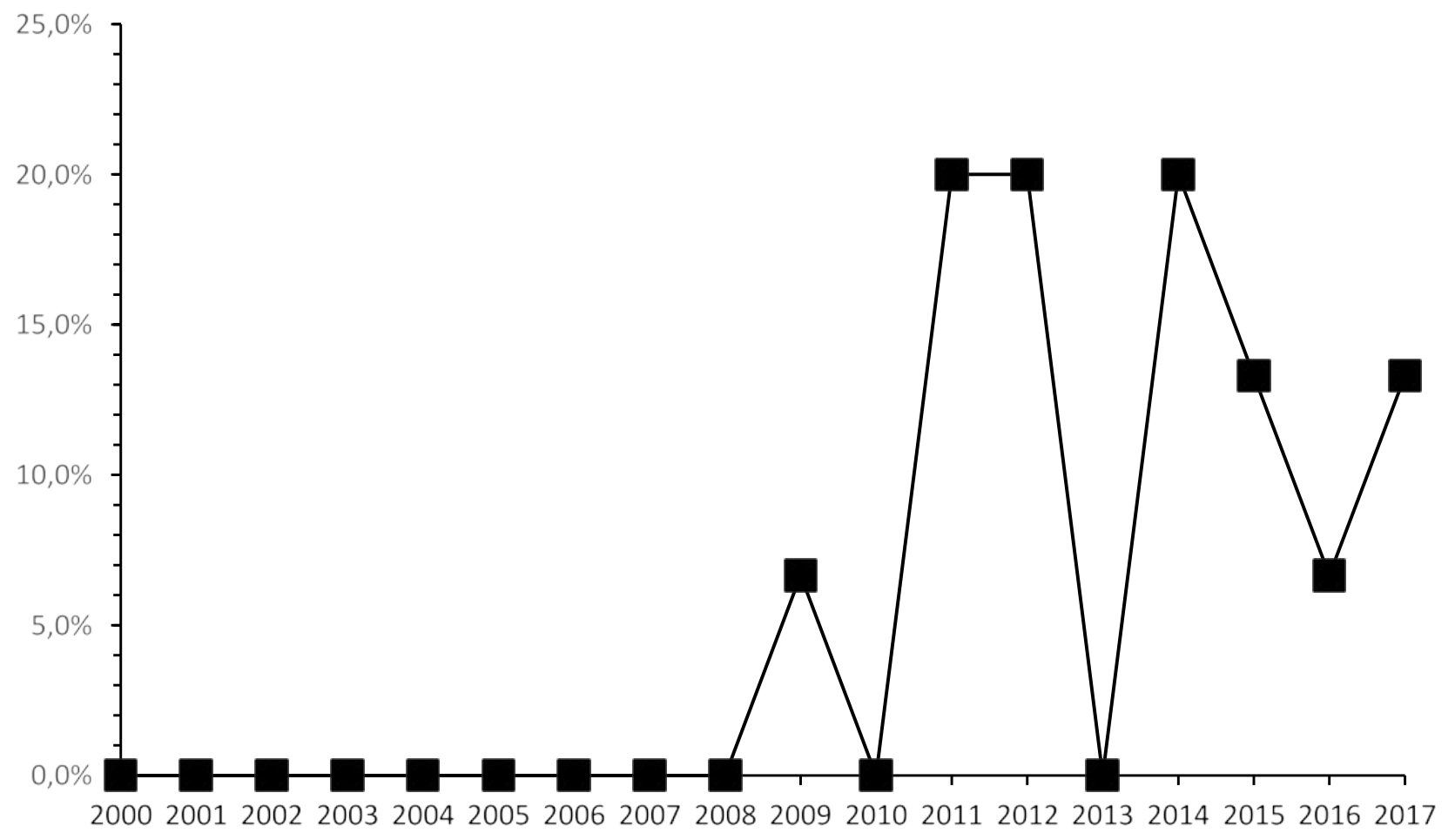

Figura 2

Distribuição em percentual e ano da frequência de produção científica. 
No Quadro 1 são apresentados os autores, título, ano de publicação, instituição e objetivos de cada estudo analisado. Portanto, foi possível identificar que, quanto aos temas estudados, seis pesquisas abordaram a sexualidade através das experiências sexuais, dos mitos e relacionamentos (Gilbert, Clark, \& Anderson, 2012; Gomez, 2011; Joharchi \& Clark, 2014; Jhai, 2017; Lehane, Dammeyer, Hovaldt, \& Elsass, 2017; Wienholz,
Seidel, Michel, Haeussler-Sczepan, \& Riedel-Heller, 2016); cinco versaram sobre violência e/ou abuso sexual (Anderson \& Leigh, 2011; Anderson \& Pezzarossi, 2012, 2014; Porter \&Williams, 2011; Powers et al., 2009); três destacaram a saúde sexual (Aldana, 2012; Pacher, Costa, Nascimento, Moura, \& Passos, 2015; Umoren \& Adejumo, 2014); e uma se referiu à homossexualidade (Abreu, Silva, \& Zuchiwschi, 2015).

Quadro 1

Caracterização dos estudos selecionados.

\begin{tabular}{|c|c|c|c|c|}
\hline Autores & Título & Ano & Instituição & Objetivo \\
\hline Powers et al. & $\begin{array}{l}\text { Interpersonal } \\
\text { violence and } \\
\text { women with } \\
\text { disabilities: } \\
\text { analysis of safety } \\
\text { promoting } \\
\text { behaviors }\end{array}$ & 2009 & $\begin{array}{l}\text { Universidade Estadual } \\
\text { de Portland; Escola } \\
\text { de Enfermagem da } \\
\text { Universidade Estadual de } \\
\text { Ohio; Baylor College of } \\
\text { Medicine; Universidade de } \\
\text { Montana; Universidade do } \\
\text { Texas e Universidade de } \\
\text { Saúde e Ciências de Oregon }\end{array}$ & $\begin{array}{l}\text { Compreender os } \\
\text { comportamentos de promoção } \\
\text { da segurança utilizados por } \\
\text { mulheres com deficiência, } \\
\text { incluindo os comportamentos } \\
\text { de segurança relacionados } \\
\text { a experiência de abuso das } \\
\text { mulheres e as características de } \\
\text { seus perpetradores. }\end{array}$ \\
\hline Porter \&Williams & $\begin{array}{l}\text { Intimate } \\
\text { violence among } \\
\text { underrepresented } \\
\text { groups on a } \\
\text { college campus }\end{array}$ & 2011 & $\begin{array}{l}\text { Instituto Rochester de } \\
\text { Tecnologia }\end{array}$ & $\begin{array}{l}\text { Investigar a incidência de } \\
\text { violência sexual, abuso físico e } \\
\text { psicológico entre grupos sub- } \\
\text { representados. }\end{array}$ \\
\hline $\begin{array}{l}\text { Anderson \& } \\
\text { Leigh }\end{array}$ & $\begin{array}{l}\text { Intimate partner } \\
\text { violence against } \\
\text { deaf female } \\
\text { college students }\end{array}$ & 2011 & Universidade Gallaudet & $\begin{array}{l}\text { Verificar a prevalência da } \\
\text { vitimização da violência de } \\
\text { parceiro íntimo em uma amostra } \\
\text { de estudantes universitárias } \\
\text { surdas. }\end{array}$ \\
\hline Gomez & $\begin{array}{l}\text { Sexual behavior } \\
\text { among Filipino } \\
\text { high school } \\
\text { students who are } \\
\text { deaf }\end{array}$ & 2011 & Universidade das Filipinas & $\begin{array}{l}\text { Investigar como adolescentes } \\
\text { filipinos surdos entenderam o } \\
\text { sexo, os fatores que os levaram } \\
\text { a suas atividades sexuais e sua } \\
\text { natureza. }\end{array}$ \\
\hline Aldana & $\begin{array}{l}\text { Representaciones } \\
\text { sociales de la } \\
\text { salud sexual de } \\
\text { adolescentes } \\
\text { sordos y oyentes } \\
\text { en la ciudad de } \\
\text { Bogotá }\end{array}$ & 2012 & $\begin{array}{l}\text { Universidad Nacional de } \\
\text { Colombia }\end{array}$ & $\begin{array}{l}\text { Descobrir e analisar a estrutura } \\
\text { e o conteúdo das representações } \\
\text { sociais da saúde sexual em três } \\
\text { grupos de adolescentes: surdos } \\
\text { usuários da língua de sinais } \\
\text { colombiana (LSC), ouvintes e } \\
\text { surdos usuários do castelhano } \\
\text { oral. }\end{array}$ \\
\hline $\begin{array}{l}\text { Anderson \& } \\
\text { Pezzarossi }\end{array}$ & $\begin{array}{l}\text { Is it abuse? } \\
\text { Deaf female } \\
\text { undergraduates' } \\
\text { labeling of } \\
\text { partner violence }\end{array}$ & 2012 & Universidade Gallaudet & $\begin{array}{l}\text { Investigar a rotulagem das } \\
\text { experiências de abuso entre } \\
\text { estudantes surdos de graduação. }\end{array}$ \\
\hline
\end{tabular}


...continuação

\begin{tabular}{|c|c|c|c|c|}
\hline Autores & Título & Ano & Instituição & Objetivo \\
\hline $\begin{array}{l}\text { Gilbert \& } \\
\text { Anderson }\end{array}$ & $\begin{array}{l}\text { Do deaf } \\
\text { individuals' } \\
\text { dating scripts } \\
\text { follow the } \\
\text { traditional } \\
\text { sexual script? }\end{array}$ & 2012 & Universidade Gallaudet & $\begin{array}{l}\text { Investigar as expectativas } \\
\text { de encontros dos indivíduos } \\
\text { surdos. }\end{array}$ \\
\hline $\begin{array}{l}\text { Anderson \& } \\
\text { Pezzarossi }\end{array}$ & $\begin{array}{l}\text { Violence against } \\
\text { deaf women: } \\
\text { Effect of partner } \\
\text { hearing status }\end{array}$ & 2014 & Universidade Gallaudet & $\begin{array}{l}\text { Investigar a prevalência, os } \\
\text { correlatos e as características } \\
\text { da vitimização da violência } \\
\text { de parceiro íntimo em } \\
\text { relacionamentos auditivos- } \\
\text { surdos e surdos-surdos. }\end{array}$ \\
\hline Joharchi \& Clark & $\begin{array}{l}\text { A glimpse } \\
\text { at American } \\
\text { deaf women's } \\
\text { sexuality }\end{array}$ & 2014 & Universidade Gallaudet & $\begin{array}{l}\text { Investigar a generatividade } \\
\text { e a sexualidade de mulheres } \\
\text { surdas. }\end{array}$ \\
\hline $\begin{array}{l}\text { Umoren \& } \\
\text { Adejumo }\end{array}$ & $\begin{array}{l}\text { Role of sexual } \\
\text { risk behaviors } \\
\text { and sexual } \\
\text { attitude in } \\
\text { perceived HIV } \\
\text { vulnerability } \\
\text { among youths } \\
\text { with disabilities } \\
\text { in two Nigerian } \\
\text { cities }\end{array}$ & 2014 & Universidade de Ibadan & $\begin{array}{l}\text { Investigar o papel da atitude } \\
\text { sexual e do comportamento } \\
\text { sexual de risco na percepção } \\
\text { da vulnerabilidade ao HIV e } \\
\text { diferenças entre jovens com } \\
\text { deficiência e jovens saudáveis. }\end{array}$ \\
\hline Abreu et al. & $\begin{array}{l}\text { Surdos e } \\
\text { homossexuais: } \\
\text { a (des)coberta } \\
\text { de trajetórias } \\
\text { silenciadas }\end{array}$ & 2015 & Universidade de Brasília & $\begin{array}{l}\text { Investigar as vivências } \\
\text { socioculturais de surdos } \\
\text { homossexuais, tendo como } \\
\text { foco para construção dos } \\
\text { dados empíricos a composição } \\
\text { de narrativas pelos sujeitos } \\
\text { pesquisados. }\end{array}$ \\
\hline Pacher et al. & $\begin{array}{l}\text { Hepatitis B and } \\
\text { C in a Brazilian } \\
\text { deaf community }\end{array}$ & 2015 & $\begin{array}{l}\text { Universidade de São Paulo } \\
\text { e Pontifícia Universidade } \\
\text { Católica de São Paulo }\end{array}$ & Não foi informado. \\
\hline Wienholz et al. & $\begin{array}{l}\text { Sexual } \\
\text { experiences of } \\
\text { adolescents with } \\
\text { and without } \\
\text { disabilities: } \\
\text { results from a } \\
\text { cross-sectional } \\
\text { study }\end{array}$ & 2016 & $\begin{array}{l}\text { Universidade de Leipzig e } \\
\text { Universidade de Ciências } \\
\text { Aplicadas }\end{array}$ & $\begin{array}{l}\text { Analisar as experiências sexuais } \\
\text { de adolescentes com e sem } \\
\text { deficiência nas atividades } \\
\text { sexuais típicas da juventude } \\
\text { (beijando, tocando genitais, } \\
\text { relações sexuais) em associação } \\
\text { a atitudes específicas e relações } \\
\text { sociais para esclarecer a } \\
\text { importância da educação } \\
\text { sexual para estudantes com e } \\
\text { sem deficiência envolvida em } \\
\text { bons conceitos das escolas } \\
\text { para pessoas com necessidades } \\
\text { especiais. }\end{array}$ \\
\hline
\end{tabular}


...continuação

\begin{tabular}{lllll}
\hline Autores & Título & Ano & Instituição & Objetivo \\
\hline Lehane et al. & $\begin{array}{l}\text { Sexuality and } \\
\text { well-being among } \\
\text { couples living } \\
\text { with acquired } \\
\text { deafblindness }\end{array}$ & 2017 & $\begin{array}{l}\text { Universidade de } \\
\text { Copenhague }\end{array}$ & $\begin{array}{l}\text { Investigar a sexualidade entre os } \\
\text { casais surdo-cegos na Dinamarca } \\
\text { e sua associação com o bem- } \\
\text { estar psicológico. }\end{array}$ \\
$\begin{array}{l}\text { Impact of } \\
\text { J romantic } \\
\text { relationships } \\
\text { counseling group } \\
\text { project on deaf } \\
\text { male adolescents } \\
\text { in a deaf school }\end{array}$ & 2017 & & $\begin{array}{l}\text { Universidade Nacional de } \\
\text { Tainan }\end{array}$ & $\begin{array}{l}\text { Discutir as relações românticas } \\
\text { de adolescentes surdos do sexo } \\
\text { masculino em uma escola surda, } \\
\text { e entender os impactos de um } \\
\text { projeto de aconselhamento } \\
\text { grupal. }\end{array}$ \\
\hline
\end{tabular}

Em relação aos artigos que abordaram a sexualidade através das experiências sexuais, dos mitos e relacionamentos, observou-se um estudo (Joharchi \& Clark, 2014) com mulheres acima dos 40 anos de idade, dois estudos (Lehane et al., 2017; Wienholz et al., 2016) que se referiram a surdez atrelada a outras deficiências (pessoas com surdocegueira e adolescentes com deficiência física, visual e auditiva), e três artigos tiveram como amostra jovens surdos (Gilbert et al., 2012; Gomez, 2011; Jhai, 2017).

No estudo de Joharchi e Clark (2014) foi identificado que as participantes relataram alta satisfação com a sexualidade, forte comunicação com o parceiro, atitudes positivas para sua própria sexualidade e apresentaram baixo índice nos itens referentes ao que falta na vida sexual atual. Os temas mais abrangentes identificados no estudo foram: a) o crescimento, constituído pelos subtemas aprendizagem com relações anteriores, resiliência adquirida nestas, generatividade, conhecimento do parceiro e percepção de ligação com ele como um todo; b) o tema da mulher, relacionado com espiritualidade e intuição, confiança ou falta dela, compromisso, infidelidade da participante e do parceiro; c) aspectos negativos da sexualidade, compreendendo a preocupação do parceiro, violência por parceiro íntimo, cultura surda e a vida bicultural; e, por fim, d) o tema denominado ser surdo, que engloba o surdo versus o parceiro ouvinte, intimidade através dos quatro sentidos e a comunicação visual durante o sexo.

No trabalho de Lehane et al. (2017), acerca da sexualidade e bem-estar de casais maiores de 50 anos de idade que convivem com a surdocegueira adquirida, foi observado que $51,1 \%$ dos participantes têm vida sexual ativa, $42,2 \%$ não têm atividade sexual, $55 \%$ relataram redução do desejo sexual, e 8,9\% informaram não ter perda desse desejo. Em relação à vida sexual $22,2 \%$ afirmaram ter algum nível de insatisfação, $20 \%$ expressaram não estar satisfeitos nem insatisfeitos, e $42,2 \%$ atestaram estar satisfeitos. Não foi evidenciada associação entre a atividade sexual e o grau de perda da visão, audição e o uso de aparelho auditivo. Quanto ao bem-estar psicológico, foi observado que $27 \%$ dos parceiros e $24 \%$ dos participantes com surdocegueira têm déficit nesse construto.

Wienholtz et al. (2016) observaram em seu estudo que as meninas sem deficiência relataram ter experimentado os três tipos de atividade sexual (beijo, toque genital e relação sexual) com maior frequência do que as que são deficientes visuais, auditivas ou físicas. Além disso, percebeu-se que os adolescentes do sexo masculino deficientes tiveram menos experiências de beijar e tocar a genitália do que os que não são deficientes. No entanto, os meninos deficientes com idade entre 14 e 16 anos apresentaram maior frequência de relações sexuais do que os não deficientes. Adicionalmente, foi constatado que mais de um terço $(36 \%)$ da amostra tinha relacionamento estável. Foi evidenciado que as meninas relatavam com maior frequência estar em relacionamento estável (44\%) do que os meninos (30\%). Observou-se que os adolescentes maiores de 15 anos de idade relataram que estavam em um relacionamento mais frequentemente (42\%) do que os mais jovens (29\%).

Na pesquisa de Gomez (2011) foi avaliado o comportamento sexual de estudantes surdos filipinos e percebeu-se que estes tinham vida sexual ativa variando de masturbação a relações sexuais completas (englobam estimulação da cabeça aos pés ou ter relações sexuais). Vale ressaltar que, quando 
indagados quanto aos fatores que influenciaram o início das suas atividades sexuais, alguns estudantes informaram ter sofrido abuso sexual por um parente ou por um adulto do mesmo sexo e relataram que este abuso foi o motivo do início de suas atividades sexuais. Esta associação pode ter ocorrido pelo fato de os participantes salientarem que não sabiam que tinham sido abusados, além de afirmarem se sentir mal quando experimentaram sexo pela primeira vez. Adicionalmente, ao serem indagados acerca das pessoas com quem fariam sexo, os participantes mencionaram primos, melhores amigos e namorados.

Em estudo acerca do impacto de um grupo de aconselhamento sobre relacionamentos românticos para adolescentes surdos no ambiente escolar, Jhai (2017) observou que inicialmente os frequentadores do grupo demonstraram dificuldade de expressar seus sentimentos mesmo que soubessem se comunicar por língua de sinais. Além disso, os professores dos participantes informaram que os adolescentes tinham dificuldade de se comunicar com os familiares e apresentavam déficits de interação social e do vocabulário emocional. Após a participação no grupo, os membros compreenderam que os relacionamentos românticos incluem aspectos de amor, gênero e casamento e identificaram mudanças positivas nas amizades, nos relacionamentos românticos e na relação professor-aluno. Dos adolescentes que estavam em uma relação durante o grupo, três foram capazes de identificar as adequações nas suas interações românticas. Destarte, esses adolescentes informaram que o aconselhamento os tornou capazes de entender o carinho e o respeito à parceira e de interagir melhor com ela.

Gilbert et al. (2012) encontraram temas diversos (conhecer amigos, familiares e lugares públicos; jantar - cena de atividade; atividades em grupo; beijo de boa-noite e desenvolvimento no relacionamento) para as três cenas de namoro, totalizando em 59 temas diferentes e que variaram de acordo com o gênero dos participantes. Esta pesquisa evidenciou que os participantes surdos pertencentes a uma família surda tinham acesso total à linguagem visual, e isso favorecia a aquisição de conhecimento sobre a sexualidade/educação sexual. No entanto, os surdos que convivem com famílias de ouvintes tenderam a não ter acesso total às informações contidas nas conversas entre seus familiares, mesmo que algum membro da família sinalizasse para o surdo.
Entre os trabalhos que abordaram violência e/ ou abuso sexual, três artigos (Anderson \& Leigh, 2011; Anderson \& Pezzarossi, 2012, 2014) tiveram como amostra estudantes de graduação do sexo feminino; um estudo (Porter \& Williams, 2011) teve como participantes homens e mulheres, homossexuais ou de outra orientação sexual, que eram discentes de graduação; e uma pesquisa (Powers et al., 2009) ocorreu fora do ambiente educacional, com mulheres adultas.

Anderson e Leigh (2011) identificaram que no ano de 2010 as participantes vivenciaram uma média de 20,65 agressões psicológicas, que foram acompanhadas por insultos, pertences destruídos e ameaças. Em relação à coerção sexual houve uma média de 8,51, incluindo ser coagido, suportar ameaças verbais e ser fisicamente forçado ao ato sexual. A agressão física teve uma média de 7,67, caracterizada por tapas, socos, chutes e queimaduras. Além desses dados, foram contabilizados 52 estudantes que sofreram um ataque físico, 22 que foram lesionadas e 61 vítimas de coerção sexual. Observou-se que as discentes surdas tiveram o dobro de vitimização no ano de 2010 quando comparadas com a amostra de estudantes ouvintes.

Foi possível verificar na pesquisa de Anderson e Pezzarossi (2012) quatro categorias: a) agressões psicológicas: a maioria da amostra vivenciou esse tipo de agressão, mais de $75 \%$ das participantes não rotularam essas agressões como abuso; b) agressões físicas: $40 \%$ da amostra relatou pelo menos um incidente; c) lesão: $19 \%$ das participantes foram feridas pelos seus parceiros, e cinco sofreram uma lesão grave; e d) coerção sexual: mais da metade da amostra sofreu esse tipo de abuso. Em outro estudo, Anderson e Pezzarossi (2014) investigaram a relação surdo-ouvinte e surdo-surdo, e perceberam que não há diferença significativa na prevalência das agressões psicológicas e físicas e de lesão quando comparado o status auditivo do parceiro. Entretanto, as mulheres com parceiros surdos eram 6,6 vezes mais propensas a reportar coerção sexual, ao passo que as mulheres com parceiros com deficiência auditiva eram 4,6 vezes mais prováveis.

Porter e Williams (2011) constataram, através da análise de regressão logística binária, diferença estatisticamente significativa entre membros de grupos sub-representados e grupos majoritários. O estudo encontrou associações entre alunos surdos e com dificuldades auditivas e estudantes que eram gays, lésbicas, bissexuais ou de outra orientação sexual 
com abuso psicológico e físico. Discentes heterossexuais do sexo feminino eram mais vulneráveis a tentativa de violação.

Na pesquisa de Powers et al. (2009) foi percebido que $92 \%$ das mulheres afirmaram ter alguém de confiança para procurar quando sofressem abuso. As participantes relataram altos níveis de segurança em relação ao dinheiro e de conhecimento legais contra os comportamentos abusivos. $\mathrm{O}$ apoio de relacionamentos, planejamento para emergências, medidas legais e a segurança nas relações de assistência pessoal foram comportamentos de promoção de segurança realizados por mulheres vítimas de abuso.

No que se refere a publicações que discutiram saúde sexual, um artigo (Aldana, 2012) relacionou este assunto com a teoria das representações sociais e teve como amostra três grupos de adolescentes (surdos usuários da língua de sinais colombiana, ouvintes e surdos usuários do castelhano oral); um estudo (Umoren \& Adejumo, 2014) teve como participantes jovens surdos, cegos e deficientes físicos, que foram relacionados com jovens sem deficiências; e no estudo de Pacher et al. (2015) todos os participantes eram surdos.

No estudo de Aldana (2012) foi possível perceber que os adolescentes surdos, fluentes na língua de sinais colombiana, tinham as seguintes representações: cuidado com o corpo, doença e prazer sexual. Já os ouvintes tinham a prevenção contra infecções sexualmente transmissíveis e o amor afetivo como representações. Por fim, os adolescentes surdos que utilizavam a língua castelhana oral tinham a educação, o amor afetivo e as relações convencionais como suas representações. Além disso, foi salientada a importância de compreender as representações sociais dos jovens surdos, pois isso auxiliará na identificação das possíveis necessidades e na execução das contribuições para essa população.

Umoren e Adejumo (2014) identificaram que o comportamento de risco sexual está relacionado e tem influência sobre a percepção de vulnerabilidade ao HIV em grupos de deficientes físicos, auditivos e visuais, enquanto a atitude sexual não tem. Os participantes com deficiência visual perceberam um maior nível de vulnerabilidade ao HIV em comparação com aqueles com deficiência auditiva e física. $\mathrm{Na}$ pesquisa de Pacher et al. (2015) foi destacada a ausência de estudos epidemiológicos sobre hepatite B e C em surdos, o que contribui para o desconhecimento desta população e para o acesso limitado a informações e a serviços de saúde.

No artigo acerca de pessoas surdas homossexuais, Abreu et al. (2015) perceberam que dois dos participantes, quando eram menores de idade, vivenciaram relação sexual de forma abusiva, pois não estavam cientes desse tipo de relação. Um dos participantes se referiu a uma situação de estupro dentro da instituição de ensino. Para a amostra há uma relação do primeiro ato sexual com a vivência atual da homossexualidade. Também foi relatado por um dos participantes que há falhas no repasse de informações para surdos, principalmente sobre o tema da sexualidade. O estudo apontou que a homossexualidade de pessoas surdas ainda não é compreendida pela sociedade, que é permeada de dúvidas, preconceitos e mitos sobre experiências afetivo-eróticas.

\section{Discussão}

Os artigos científicos encontrados não foram distribuídos de forma igualitária no período delimitado para a busca (2000-2017). Adicionalmente, os anos de 2000 a 2008, 2010 e 2013 não tiveram publicações.

A lacuna de produção científica pode estar relacionada à proibição do uso da língua de sinais no ano de 1880, após o II Congresso de Educação de Surdos ocorrido em Milão. Além disso, por cerca de cem anos, o oralismo foi instituído como método de ensino obrigatório para surdos. No entanto, a partir de 1960 os estudos passaram a se voltar para os diferentes métodos de ensino (língua oral, língua de sinais, comunicação total e bilinguismo), a diferença cultural entre surdos e ouvintes, a cognição e as relações familiares, o que pode ter suprimido estudos com o foco em outros aspectos relacionados à surdez (Bisol, 2008; Carvalho, 2007; Lane, 1997; Lebedeff, 2010).

A associação de estudos sobre a surdez com os de outras deficiências (Lehane et al., 2017; Umoren \& Adejumo, 2014; Wienholz et al., 2016) pode estar relacionada com o modelo clínico que percebe a surdez como deficiência, o que subsidia a ideia de incapacidade e de anormalidade dos surdos e os torna dependentes dos ouvintes (Marostega \& Santos, 2006; Strobel, 2008). Em contrapartida, foram encontradas diversas pesquisas (Abreu et al., 2015; Aldana, 2012; Anderson \& Leigh, 2011; Anderson \& Pezzarossi, 2012, 2014; Gilbert et al., 2012; Gomez, 2011; Jhai, 2017; Joharchi \& Clark, 2014; Pacher et al., 2015; Porter \& Williams; 2011; Powers et al., 2009) apenas 
com participantes surdos, e isto pode estar concatenado com o modelo socioantropológico, que percebe a surdez como uma diferença linguística. É notório que esses dois modelos continuam presentes na atualidade e exercem influência no modo de instituições de ensino, familiares e a sociedade perceberem e lidarem com os surdos (Bisol \& Sperb, 2010; Skliar, 2016).

Pesquisas (Bento \& Bueno, 2005; Flinn, 1982; Getch et al., 2001; Trejo, 2005) corroboraram os achados de estudos que se basearam nas experiências sexuais, nos mitos e relacionamentos e na saúde sexual, ao salientarem a importância do comportamento crítico e reflexivo dos surdos para a construção do conhecimento sobre a sexualidade. Para isso, essas pesquisas enfatizaram a necessidade de abordar o tema da sexualidade nas escolas e com a família dos surdos. Foram sugeridas a organização de eventos, novas pesquisas e um programa de treinamento sobre a educação sexual para os professores que lecionam a surdos. Este programa possibilitaria que o indivíduo faça escolhas apropriadas para a sua saúde sexual e interpessoal. Também foi evidenciado que a escola é um local de comunicação direta que contribui para a diminuição de mitos e estereótipos acerca da sexualidade.

Em relação aos artigos que abordaram violência e/ou abuso sexual, foi possível perceber no estudo de Anderson e Leigh (2011) que 91 dos 100 participantes foram vítimas de pelo menos uma agressão psicológica e que as estudantes surdas relataram maior agressão psicológica do que o grupo de ouvintes. Tais dados assemelham-se aos apresentados em outros estudos (Costa, Serafim, \& Nascimento, 2015; Rangel \& Oliveira, 2010), em que a violência mais prevalente e com maior continuidade foi a psicológica. Ademais, foi evidenciado que esta violência é menos percebida pela sociedade, pois os meios de comunicação dão ênfase apenas à violência sexual.

Com os resultados compilados neste estudo sobre o tema da violência, indentificou-se também maior número de pesquisas (Anderson \& Leigh, 2011; Anderson \& Pezzarossi, 2012, 2014; Powers et al., 2009) voltadas a mulheres. Entretanto, foi encontrado um estudo (Porter \& Williams; 2011) em que houve a participação de homens e mulheres classificados como minorias em cursos de graduação (estudantes surdos, deficientes auditivos, homossexuais, bisexuais e membros de minorias raciais e étnicas), embora estes grupos não tenham sido predominantes nos artigos científicos encontrados. Os dados apresentados estão respaldados no fato de a mulher ser considerada a principal vítima da violência de gênero e este assunto ser apontado como um problema mundial (Brasil, 2007).

Embora existam mais estudos voltados para mulheres, Brownridge (2009) aponta a necessidade de aumentar o número de pesquisas com mulheres com deficiência e mulheres surdas no intuito de prevenir os modos de violência. Adicionalmente, Porter e Williams (2011) salientam a importância de profissionais de saúde expandirem as orientações sobre violência sexual para além do público feminino, pelo fato de os grupos supracitados também serem vítimas desse tipo de violência.

Em relação à violência contra a mulher, Anderson e Pezzarossi (2012) observaram em seu estudo que a maior parte da amostra preferiu não rotular como violência os comportamentos realizados pelos seus parceiros. Estes dados são similares aos de pesquisas brasileiras (Albuquerque Netto, Moura, Silva, Penna, \& Pereira, 2015; Brandão, 1998; Jong, Sadala, \& Tanaka, 2008; Schraiber \& d'Oliveira, 1999; Schraiber, d'Oliveira, França-Júnior, \& Pinho, 2002) que salientaram as subnotificações desta violência no país. Ademais, foi constatado que a busca de auxílio psicossocial ocorre quando as mulheres vítimas da violência se sentem no limite da tolerância.

Nos resultados obtidos nesta pesquisa também se percebeu a relação entre os estudos de Gomez (2011) e Abreu et al. (2015) quando evidenciaram o abuso sexual como elementar no aumento do comportamento sexual dos jovens. Ademais, esses estudos assinalaram que os abusos sexuais geralmente ocorrem no ambiente familiar e que a maior parte dos jovens do sexo masculino é abusada por pessoas do mesmo sexo. Devido ao fato de abusos sexuais ocorrerem em ambientes familiares e por serem suprimidos, estudos (Florentino, 2015; Prado, 2004; Romaro \& Capitão, 2007; Silva, 2000) relatam a necessidade de profissionais estarem atentos e preparados para identificar e intervir nesses casos, pois as consequências dos abusos na vida do indivíduo são distintas (físicas, psíquicas e sociais), classificadas como graves e deixam marcas.

No que se refere a homossexualidade e surdez, dos 15 artigos encontrados, um abordou o tema (Abreu et al., 2015) e salientou o duplo preconceito (ser surdo e homossexual) proveniente dos próprios familiares e da sociedade. Por esse fato, alguns surdos escolhem omitir sua homossexualidade para se proteger dos estigmas sociais. No entanto, no estudo de 
Swarts (1995), com ouvintes e surdos homossexuais, foi percebido que os surdos revelaram sua homossexualidade perante a sociedade e se apresentaram mais satisfeitos com suas relações amorosas do que os homessexuais ouvintes.

Apesar desses dois estudos terem aproximadamente duas décadas de diferença, há maneiras distintas de surdos homossexuais lidarem com os familiares e a sociedade. Isso pode estar relacionado com as distinções entre as culturas $\square$ já que as pesquisas foram realizadas em diferentes países $\otimes$, que influenciam na forma de perceber o outro. Outrossim, um ponto em comum das duas pesquisas foi a presença do preconceito dos familiares para com o surdo. Adicionalmente, na pesquisa de Swarts (1995), os surdos de famílias surdas perceberam o vínculo familiar enfraquecido por conta de sua orientação sexual.

Os resultados do presente estudo evidenciaram a escassez de publicações de artigos científicos referentes à sexualidade de surdos. Dados semelhantes foram encontrados na pesquisa de Bento e Bueno (2005), ao afirmarem que a escassez de pesquisas acerca da sexualidade de surdos promove a negligência da temática, a restrição de informações e a exclusão dessas pessoas. Neste sentido, Getch et al. (2001) ratificam os estudos supracitados ao destacarem a importância da disseminação da temática em questão e da abrangência para todas as culturas e níveis socais. Adicionalmente, Maia (2006) reitera que a sexualidade precisa ser debatida e dismistificada na sociedade.

\section{Considerações finais}

Com relação ao objetivo desta revisão, foi possível identificar os artigos científicos produzidos no Brasil e em outros países sobre a sexualidade da pessoa surda. Assim, percebeu-se a escassez de produções científicas referentes a essa temática. Além disso, foram identificados os temas abordados nos estudos, e os mais frequentes foram a sexualidade através das experiências sexuais, dos mitos e relacionamentos e a violência e/ou abuso sexual. Cabe ressaltar que o assunto com menor produção científica foi a homossexualidade, com somente um artigo.

De modo geral, os estudos analisados abrangeram os diversos aspectos da sexualidade e não se limitaram à saúde sexual. Foi notória a preocupação com os valores, a afetividade, o bem-estar e a satisfação dos surdos em seus relacionamentos e atividade sexual. Adicionalmente, observou-se a presença do modelo clínico e socioantropológico e o modo como esses determinam as percepções de pesquisadores, educadores, familiares e da sociedade em relação a surdos. Ademais, há um consenso entre os estudos sobre a relevância de difundir a temática da sexualidade em todas as culturas e níveis sociais, independentemente do modelo a ser escolhido. Salienta-se também a necessidade de a escola e familiares participarem da educação sexual dos surdos.

Espera-se com essa revisão sistemática motivar os profissionais de diversas áreas, e principalmente educadores, psicólogos e profissionais da saúde, a pesquisar e atuar com os surdos para desconstruir os mitos existentes sobre o assunto, debater a sexualidade de forma abrangente e dirimir os comportamentos de risco e a vulnerabilidade a violência e/ou abuso sexual destes. Outrossim, ressalta-se a importância de os autores brasileiros buscarem diversificar os temas relacionados à sexualidade para que os dados obtidos possam subsidiar as futuras práticas desses profissionais e dos familiares.

Embora tenham sido encontrados poucos estudos, salienta-se a importância dos dados obtidos para futuras pesquisas e para a sociedade em geral. Além disso, sugerem-se novas publicações que deem continuidade à amplitude conceitual da sexualidade e da diversidade sexual com a participação das pessoas surdas no intuito de compreendê-las, orientá-las e informá-las sobre a temática em questão. As principais limitações deste estudo foram o bloqueio de acesso a artigos e a existência de artigos com palavras-chave, objetivos e resumos inexplícitos. Apesar de o período de delimitação dos estudos ter sido substancial (20002017), a extensão do período pôde oferecer a inserção de estudos relevantes sobre a presente temática.

\section{Referências}

Abreu, F. S. D., Silva, D. N. H., \&Zuchiwschi, J. (2015). Surdos e homossexuais: A (des) coberta de trajetórias silenciadas. Temas em Psicologia, 23(3), 607-620. https:// doi.org/10.9788/TP2015.3-07

Albuquerque Netto, L. A., Moura, M. A. V., Silva, G. F., Penna, L. H. G., \& Pereira, A. L. F. (2015). Mulheres em situação de violência pelo parceiro íntimo: tomada de decisão por apoio institucional especializado. Revista Gaúcha de Enfermagem, 36(esp), 135-42. https://doi.org/10.1590/1983- 1447.2015.esp.54361 
Aldana, J. C. (2012). Representaciones sociales de la salud sexual de adolescentes sordos y oyentes en la ciudad de Bogotá. Pensamiento Psicológico, 10(2), 35-47. http://www.scielo.org.co/pdf/pepsi/v10n2/v10n2a05.pdf

Anderson, M. L., \& Leigh, I. W. (2011). Intimate partner violence against deaf female college students. Violence Against Women, 17(7) 822-834. https:// doi.org/10.1177/1077801211412544

Anderson, M. L., \& Pezzarossi, C. M. K. (2012). Is it abuse? Deaf female undergraduates' labeling of partner violence. Journal of Deaf Studies and Deaf Education, 17(2), 273-286. https://doi.org/10.1093/deafed/enr048

Anderson, M. L., \& Pezzarossi, C. M. K. (2014). Violence against deaf women: Effect of partner hearing status. Journal of Deaf Studies and Deaf Education, 19(3), 411-421. https://doi.org/10.1093/deafed/ent053

Aquino, J. G. (Org.). (1997). Sexualidade na escola: Alternativas teóricas e práticas. São Paulo, SP: Summus.

Baker-Duncan, N., Dancer, J., Gentry, B., Highly, P., \& Gibson, B. (1997). Deaf adolescents' knowledge of aids: Grade and gender effects. American Annals of the Deaf, 142(5), 368-372. https://doi.org/10.1353/aad.2012.0344

Bandarra, A. J. E. (2013). No xadrez das sexualidades: conhecimentos, atitudes e comportamentos de jovens adolescentes surdos face às ISTS (Dissertação de mestrado). Instituto de Psicologia e Ciências da Educação, Universidade Lusíada do Porto, Porto.

Batista, T. P. M., \& Reis, J. G. (2011). A família de estudantes surdos e a importância da comunicação em Libras para processo de aprendizagem. Anais do Encontro da Associação Brasileira de Pesquisadores em Educação Especial, 7, 1201-1213. http://www.uel.br/eventos/congressomultidisciplinar/pages/arquivos/anais/2011/ familia/113-2011.pdf.

Bento, I. C. B. (2005). Educação preventiva em sexualidade, istlaids para o surdo através da pesquisalação (Tese de doutorado). Escola de Enfermagem de Riberão Preto, Universidade de São Paulo, Riberão Preto, SP.

Bento, I. C. B., \& Bueno, S. M. V. (2005). A aids sob a ótica do surdo adulto jovem. DST: Jornal Brasileiro de Doenças Sexualmente Transmissiveis, 17(4), 288-294. http://www.dst.uff.br/revista17-4-2005/A-Aids-Sob-a-Etica.pdf

Bisol, C. A. (2008). Adolescer no contexto da surdez: questões sobre a sexualidade (Tese de doutorado). Universidade Federal do Rio Grande do Sul, RS.

Bisol, C. A., \& Sperb, T. M. (2010). Discursos sobre a surdez: Deficiência, diferença, singularidade e construção de sentido. Psicologia: Teoria e Pesquisa, 26(1), 7-13. https://doi.org/10.1590/S0102-37722010000100002

Bisol, C. A., Sperb, T. M., Brewer, T. H., Kato, S. K., \& Shor-Posner, G. (2008). HIV/aids knowledge and health-related attitudes and behaviors among deaf and hearing adolescents in Southern Brazil. American Annals of the Deaf, 153(4), 349-356. https://doi.org/10.1353/aad.0.0055

Brandão, E. R. (1998). Violência conjugal e o recurso feminino à polícia. In C. Bruschini, \& H. B. Hollanda (Orgs.), Horizontes plurais: novos estudos de gênero no Brasil (pp. 51-84). São Paulo, SP: Fundação Carlos Chagas. http://www.fcc.org.br/conteudosespeciais/bibliografiaanotada/arquivo/HorizontesPlurais.pdf

Brasil. (2007). Política nacional de enfrentamento à violência contra as mulheres. Brasília, DF: Secretaria Especial de Políticas para as Mulheres. http://www.spm.gov.br/sobre/publicacoes/publicacoes/2011/politica-nacional

Brownridge, D. A. (2009). Situating research on Safety Promoting Behaviors among disabled and deaf Victims of Interpersonal Violence. Violence Against Women, 15(9), 1075-1079. https:// doi.org/10.1177/ 1077801209340311.

Carvalho, P. V. (2007). Breve história dos surdos no mundo e em Portugal. Lisboa: Surd'Universo.

Castro, M. G., Abramovay, M., \& Silva, L. B. (2004). Juventude e sexualidade. Brasília, DF: Unesco Brasil.

Chaveiro, N., Barbosa, M. A., \& Porto, C. C. (2008). Revisão de literatura sobre o atendimento ao paciente surdo pelos profissionais da saúde. Revista da Escola de Enfermagem da USP, 42(3), 578-583. https:// doi.org/10.1590/ S0080-62342008000300023

Costa, M. S., Serafim, M. L. F., \& Nascimento, A. R. S. (2015). Violência contra a mulher: descrição das denúncias em um centro de referência de atendimento à mulher de Cajazeiras, Paraíba, 2010 a 2012. Revista Epidemiologia e Serviços de Saúde, 24(3), 551-558. https://doi.org/10.5123/S1679-49742015000300022

Dizeu, L. C. T. B., \& Caporali, S. A. (2005). A língua de sinais constituindo o surdo como sujeito. Educação e Sociedade, 26(91), 583-597. https:// doi.org/10.1590/S0101-73302005000200014 
Doyle, A. G. (1995). AIDS knowledge, attitudes and behaviors among deaf college students: a preliminary study. Sexuality and Disability, 13(2), 107-134. https://doi.org/10.1007/BF02590060

Flinn, S. (1982). Preparing teachers of the deaf to teach sex education. Sexuality and Disability, 5(4), 230-236. https://doi.org/10.1007/BF01119864

Florentino, B. R. B. (2015). As possíveis consequências do abuso sexual praticado contra crianças e adolescentes. Fractal: Revista de Psicologia, 27(2), 139-144. https://doi.org/10.1590/1984-0292/805

Getch, Y. Q., Branca, D. L., Fitz-Gerald, D., \& Fitz-Gerald, M. (2001). A rationale and recommendations for sexuality education in schools for students who are deaf. American Annals of the Deaf, 146(5), 401-408. https://doi.org/10.1353/aad.2012.0209

Gilbert, G. L., Clark, M. D., \& Anderson, M. L. (2012). Do deaf individuals' dating scripts follow the traditional sexual script? Sexuality \& Culture, 16, 90-99. https://doi.org/10.1007/s12119-011-9111-4

Gomez, M. G. A. (2011). Sexual behavior among Filipino high school students who are deaf. Sexuality and Disability, 29, 301-312. https:// doi.org/10.1007/s11195-011-9215-7

Jhai, Z.-T. (2017). Impact of a romantic relationships counseling group project on deaf male adolescents in a deaf school. Sexuality and Disability, 35, 185-206. https://doi.org/10.1007/s11195-017-9481-0

Job, J. (2004). Factors involved in the ineffective dissemination of sexuality information to individuals who are deaf or hard of hearing. American Annals of the Deaf, 149(3), 264-273. https://doi.org/10.1353/aad.2004.0025

Joharchi, H. A., \& Clark, M. D. (2014). A glimpse at American deaf women's sexuality. Psychology, 5(13), 1536-1549. https://doi.org/10.4236/psych.2014.513164

Jong, L. C., Sadala, M. L. A., \& Tanaka, A. C. D. (2008). Desistindo da denúncia ao agressor: relato de mulheres vítimas de violência doméstica. Revista da Escola de Enfermagem da USP, 42(4), 744-751. https://doi.org/10.1590/ S0080-62342008000400018

Kennedy, S. G., \& Buchhoiz, C. L. (1995). HIV and AIDS among the deaf. Sexuality and Disability, 13(2), 145-158. https://doi.org/10.1007/BF02590062

LaBarre, A. (1998). Treatment of sexually abused children who are deaf. Sexuality and Disability, 16(4), 321-324. https://doi.org/10.1023/A:1023072029386

Lane, H. (1997). A máscara da benevolência: a comunidade surda amordaçada (C. Reis, Trad.). Lisboa: Instituto Piaget.

Lebedeff, T. B. (2010). Surdez e Sexualidade: uma discussão sobre a necessidade de empoderamento linguístico e acesso à informação. Anais do Encontro de Pesquisa em Educação da Região Sul-Anpedsul, 8, 1-17.

Lehane, C. M., Dammeyer, J., Hovaldt, H. B., \& Elsass, P. (2017). Sexuality and well-being among couples living with acquired deafblindness. Sexuality and Disability, 35, 135-146. https://doi.org/10.1007/s11195-016-9470-8

Luckner, J. L., \& Gonzales, B. R. (1993). What deaf and hard-of-hearing adolescents know and think about aids. American Annals of the Deaf, 138(4), 338-342. https://doi.org/10.1353/aad.2012.0390

Maia, A. C. B. (2006). Sexualidade e deficiências. São Paulo, SP: Editora Unesp.

Marin, M. C., \& Goés, R. A. (2006). Experiência de pessoas surdas em esferas de atividade do cotidiano. Caderno CEDES, 26(69), 231-249. https://doi.org/10.1590/S0101-32622006000200007

Marostega, V. L., \& Santos, A. N. (2006). A influência da comunicação que envolve família-filho-escola no processo de desenvolvimento e aprendizagem do sujeito surdo. Revista Educação Especial, (28), 1-7.

Oliveira, L. M. B. (2012). Cartilha do Censo 2010: Pessoas com Deficiência. Brasília, DF: Secretaria de Direitos Humanos da Presidência da República.

Pacher, B. M., Costa, M. R. B., Nascimento, M. M. P., Moura, M. C., \& Passos, A. D. C. (2015). Hepatitis B and C in a Brazilian deaf community. Revista da Sociedade Brasileira de Medicina Tropical, 48(5), 603-606. https://doi.org/10.1590/0037-8682-0058-2015

Porter, J., \&Williams, L. M. (2011). Intimate violence among underrepresented groups on a college campus. Journal of Interpersonal Violence, 26(16), 3210-3224. https://doi.org/10.1177/0886260510393011 
Powers, L. E., Renker, P., Robinson-Whelen, S., Oschwald, M., Hughes, R., Swank, P., \& Curry, M. A. (2009). Interpersonal violence and women with disabilities: Analysis of safety promoting behaviors. Violence Against Women, 15(9), 1040-1069. https://doi.org/10.1177/1077801209340309

Prado, M. C. C. A. (Org.). (2004). O mosaico da violência. São Paulo, SP: Vetor.

Rangel, C. M. F. R. B. A., \& Oliveira, E. L. (2010). Violência contra as mulheres: fatores precipitantes e perfil de vítimas e agressores. Anais do Seminário Internacional Fazendo Gênero: Diásporas, Diversidades, Deslocamentos, 9, 1-11. http://www.fazendogenero.ufsc.br/9/resources/anais/1277848018_ARQUIVO_fazendogenero_Celina_ Elzira.pdf

Ribeiro, K. (2011). Sexualidade e gênero: estudos das relações afetivas de jovens surdas de uma escola municipal de educação especial de São Paulo (Tese de doutorado). Universidade de São Paulo, São Paulo, SP.

Romaro, R. A., \& Capitão, C. G. (2007). As faces da violência: aproximações, pesquisas, reflexões. São Paulo, SP:Vetor.

Schraiber, L. B., \& d'Oliveira, A. F. L. P. (1999). Violência contra mulheres: interfaces com a saúde. Interface Comunicação, Saúde, Educação, 3(5), 15-26. https://doi.org/10.1590/S1414-32831999000200003

Schraiber, L. B., d'Oliveira, A. F. P. L., França-Júnior, I., \& Pinho, A. A. (2002). Violência contra a mulher: estudo em uma unidade de atenção primária à saúde. Revista Saúde Pública, 36(4), 470-477. https://doi.org/10.1590/ S0034-89102002000400013

Silva, I. R. (2000). Abuso e trauma. São Paulo, SP: Vetor.

Skliar, C. (2016). Os estudos surdos em educação: problematizando a normalidade. In C. Skiliar (Ed.), Um olhar sobre as diferenças (8a ed., pp. 7-32). Porto Alegre, RS: Mediação.

Sousa, M. J. A., \& Moleiro, C. M. M. (2015). Homens gays com deficiência congénita e/ou adquirida, física e/ou sensorial: duplo-fardo social. Sexualidad, Salud y Sociedad, (20), 72-90. https://doi.org/10.1590/1984-6487. sess.2015.20.07.a

Sousa, R. A., \& Pagliuca, L. M. F. (2001). Cartilha sobre saúde sexual e reprodutiva para surdos como tecnologia emancipatória: relato de experiência. Revista RENE, 2(2), 80-86. https://doi.org/10.15253/rev\%20rene.v2i2.5889Strobel, K. L. (2008). Surdos: vestígios culturais não registrados na história (Tese de doutorado). Universidade Federal de Santa Catarina, Florianópolis, SC.

Sullivan, P. M., \& Knutson, J. F. (1998). Maltreatment and behavioral characteristics of youth who are deaf and hard-of-hearing. Sexuality and Disability, 16(4), 295-319. https://doi.org/10.1023/A:1023019912548

Sullivan, P. M., Scanlan, J. M., Brookhouser, P. E., \& Schulte, L. E. (1992). The effects of psychotherapy on behavior problems of sexually abused deaf children. Child: Abuse \& Neglect, 16, 297-307. https:// doi.org/10.1016/0145-2134(92)90036-Q

Swartz, D. B. (1995). Cultural implications of audiological deficits on the homosexual male. Sexuality and Disability, 13(2), 159-181. https://link.springer.com/article/10.1007/BF02590063

Trejo, M. T. (2005). La construccion de la sexualidad en adolescentes sordos y sordas. Archivos Hispanoamericanos de Sexologia, 11(2), 203-218. http://www.cultura-sorda.org/wp-content/uploads/2015/03/Tellez_Construcion_ Sexualidad_adolescentes_sordos_2005.pdf

Umoren, A. M., \& Adejumo, A. O. (2014). Role of sexual risk behaviors and sexual attitude in perceived HIV vulnerability among youths with disabilities in two Nigerian cities. Sexuality and Disability, 32, 323-334. https://doi.org/10.1007/s11195-014-9366-4

Wienholz, S., Seidel, A., Michel, M., Haeussler-Sczepan, M., \& Riedel-Heller, S. G. (2016). Sexual experiences of adolescents with and without disabilities: results from a cross-sectional study. Sexuality and Disability, 34, 171-182. https://doi.org/10.1007/s11195-016-9433-0

World Health Organization. (2013). Millions of people in the world have hearing loss that can be treated or prevented. Geneva:WHO. http://www.who.int/pbd/deafness/news/Millionslivewithhearingloss.pdf?ua=1

Yu, B., Chen, J., Jin, Y., Zhang, W., Feng, Y., \& Zhao, X. (2017). The knowledge and skills related to sexual abuse prevention among Chinese children with hearing loss in Beijing. Disability and Health Journal, 10, 344-349. https:// doi.org/10.1016/j.dhjo.2016.12.014 


\section{Valéria Maria Azevedo Guimarães}

Psicóloga, Mestre e doutoranda em Psicologia pelo Programa de Pós-Graduação em Psicologia da Universidade Federal de Sergipe, São Cristóvão - SE. Brasil.

E-mail: guimaraes.psicologa@gmail.com

(D) https://orcid.org/0000-0002-3782-4973

\section{Joilson Pereira da Silva}

Psicólogo, Doutor em Psicologia pela Universidade Complutense de Madri-Espanha, Mestre em Psicologia pela Universidade Federal da Paraíba. Professor associado da Universidade Federal de Sergipe e professor credenciado do Programa de Pós-Graduação em Psicologia da Universidade Federal de Sergipe, São Cristóvão - SE. Brasil.

E-mail: joilsonp@hotmail.com

(iD) https://orcid.org/0000-0001-9149-3020

Endereço para envio de correspondência:

Universidade Federal de Sergipe. Programa de Pós-Graduação em Psicologia (PPGPSI) - Didática II - $1^{\circ}$ andar. Av. Marechal Rondon, s/n, Jardim Rosa Elze. CEP: 49100-000. São Cristóvão - Sergipe. Brasil.

Recebido 31/05/2018

Aceito 18/06/2019

Received 05/31/2018

Approved 06/18/2019

Recibido 31/05/2018

Aceptado 18/06/2019

Como citar: Guimarães, V. M. A., \& Silva, J. P. (2020). Sexualidade e Surdez: uma Revisão Sistemática. Psicologia: Ciência e Profissão, 40, 1-16. https://doi.org/10.1590/1982-3703003201645

How to cite: Guimarães, V. M. A., \& Silva, J. P. (2020). Sexuality and deafness: a systematic review. Psicologia: Ciência e Profissão, 40, 1-16. https://doi.org/10.1590/1982-3703003201645

Cómo citar: Guimarães, V. M. A., \& Silva, J. P. (2020). Sexualidad y Sordera: Una Revisión Sistemática. Psicologia: Ciência e Profissão, 40, 1-16. https://doi.org/10.1590/1982-3703003201645 